\title{
A Model of Perinatal Stress and Childhood Wheezing: ELSPAC-CZ Cohort
}

\author{
Irena Stepanikova ${ }^{1}$, Vojtech Thon ${ }^{2}$, Ondrej Mikes $^{2}$, and Jana Klanova ${ }^{2}$ \\ ${ }^{1}$ University of Alabama at Birmingham \\ ${ }^{2}$ Masaryk University
}

July 22, 2020

\begin{abstract}
Background Prenatal origins of wheezing are not fully understood. This study develops a model of mechanisms linking perinatal stress exposure to wheeze phenotypes in children. Methods Data were obtained from 1,849 mother-child dyads participating in ELSPAC-CZ birth cohort. Wheeze phenotypes assessed between birth and age seven years included "never wheeze", "earlyonset transient (EOT) wheeze," "early-onset persistent (EOP) wheeze," and "late-onset (LO) wheeze." Prenatal and postnatal maternal stress exposures were assessed in mid-pregnancy and six months post-delivery, respectively, using an inventory of 42 life events. Results In adjusted models, children in the highest tercile (high) vs. lowest tercile (low) of prenatal life events had a $44 \%$ higher risk of EOT wheeze (relative risk ratio $[\mathrm{RRR}]=1.44,95 \%$ confidence interval $[\mathrm{CI}]=1.06-1.95, \mathrm{p}=0.02$ ) and $69 \%$ higher risk of $\mathrm{LO}$ wheeze $(\mathrm{RRR}=1.69,95 \% \mathrm{CI}=1.13-2.52, \mathrm{p}=0.01)$. High vs. low exposure to postnatal life events predicted a $74 \%$ increase in the risk of EOT wheeze $(\mathrm{RRR}=1.74,95 \% \mathrm{CI}=1.27-2.38, \mathrm{p}<0.001)$ and $101 \%$ increase for $\mathrm{EOP}$ wheeze $(\mathrm{RRR}=2.01$, 95\% $\mathrm{CI}=1.23-3.26, \mathrm{p}=0.005$ ). Postnatal life events partially mediated between prenatal life events and any wheeze (high vs. low life events: indirect effect $\mathrm{OR}=1.13,95 \% \mathrm{CI}=1.06-1.21, \mathrm{p}<0.001)$. Lower respiratory tract infections and secondary smoke partially mediated between postnatal life events and any wheeze (indirect effects $\mathrm{OR}=1.06,95 \% \mathrm{CI}=1.02-1.09, \mathrm{p}=0.002$ and $\mathrm{OR}=1.02,95 \%$ CI 1.001-1.05, $\mathrm{p}=0.035$, respectively). Conclusions Exposures to prenatal and postnatal life events are risk factors for development of wheezing. Prenatal stress contributes to wheeze directly and also through postnatal life events and respiratory infections.
\end{abstract}

\section{A Model of Perinatal Stress and Childhood Wheezing: ELSPAC-CZ Cohort}

Irena Stepanikova, Ph.D., ${ }^{\mathrm{a}}$ Vojtech Thon, M.D., Ph.D., ${ }^{\mathrm{b}}$ Ondrej Mikes, Ph.D., ${ }^{\mathrm{b}}$ Jana Klanova, Ph.D. ${ }^{\mathrm{b}}$

${ }^{a}$ Department of Sociology, University of Alabama at Birmingham, Birmingham, AL, USA

b RECETOX, Faculty of Science, Masaryk University, Brno, Czech Republic

Corresponding author:

Irena Stepanikova

Department of Sociology, University of Alabama at Birmingham

1401 University Drive

Birmingham, Alabama, USA

irena@uab.edu

Tel: 650575404

Words: Abstract, 244; Body, 3425. 
Tables, 3. Figures, 3

Keywords. Gender, depressive symptoms, mental health

Financial support. The ELSPAC-CZ study is funded by the Ministry of Education, Youth and Sports of the Czech Republic and European Structural and Investment Funds (CETOCOEN PLUS project: CZ.02.1.01/0.0/0.0/15_003/0000469 and the RECETOX Research Infrastructures: LM20150518121. and CZ.02.1.01/0.0/0.0/16_013/0001761). We also acknowledge the Teaming project: CETOCOEN EXCELLENCE Teaming 2 project supported by Horizon2020 (857560) and the Czech Ministry of Education, Youth and Sports (CZ.02.1.01/0.0/0.0/18_046/0015975). The funders of the study had no role in study design, data collection, data analysis, data interpretation, or writing of the report. The views and opinions expressed in this paper are those of the authors and do not reflect the official position of any of the organizations for which the authors work.

Author contributions. Irena Stepanikova participated in all aspects of the study, including conceptualization and design, literature review, data analysis, interpretation, and writing of all sections. Ondrej Mikes participated in the statistical analyses and writing. Vojtech Thon participated in conceptualization and interpretation of results. Jana Klanova participated in acquiring and preparing the data.

Running title . Perinatal stress and childhood wheezing: ELSPAC-CZ

Abstract

Background Prenatal origins of wheezing are not fully understood. This study develops a model of mechanisms linking perinatal stress exposure to wheeze phenotypes in children.

Methods Data were obtained from 1,849 mother-child dyads participating in ELSPAC-CZ birth cohort. Wheeze phenotypes assessed between birth and age seven years included "never wheeze", "early-onset transient (EOT) wheeze," "early-onset persistent (EOP) wheeze," and "late-onset (LO) wheeze." Prenatal and postnatal maternal stress exposures were assessed in mid-pregnancy and six months post-delivery, respectively, using an inventory of 42 life events.

Results In adjusted models, children in the highest tercile (high) vs. lowest tercile (low) of prenatal life events had a $44 \%$ higher risk of EOT wheeze (relative risk ratio $[\mathrm{RRR}]=1.44,95 \%$ confidence interval $[\mathrm{CI}]=1.06-1.95, \mathrm{p}=0.02)$ and $69 \%$ higher risk of $\mathrm{LO}$ wheeze $(\mathrm{RRR}=1.69,95 \% \mathrm{CI}=1.13-2.52, \mathrm{p}=0.01)$. High vs. low exposure to postnatal life events predicted a $74 \%$ increase in the risk of EOT wheeze $(\mathrm{RRR}=1.74$, $95 \% \mathrm{CI}=1.27-2.38, \mathrm{p}<0.001)$ and $101 \%$ increase for $\mathrm{EOP}$ wheeze $(\mathrm{RRR}=2.01,95 \% \mathrm{CI}=1.23-3.26, \mathrm{p}=0.005)$. Postnatal life events partially mediated between prenatal life events and any wheeze (high vs. low life events: indirect effect $\mathrm{OR}=1.13,95 \% \mathrm{CI}=1.06-1.21, \mathrm{p}<0.001)$. Lower respiratory tract infections and secondary smoke partially mediated between postnatal life events and any wheeze (indirect effects $\mathrm{OR}=1.06,95 \%$ $\mathrm{CI}=1.02-1.09, \mathrm{p}=0.002$ and $\mathrm{OR}=1.02,95 \%$ CI 1.001-1.05, $\mathrm{p}=0.035$, respectively).

Conclusions Exposures to prenatal and postnatal life events are risk factors for development of wheezing. Prenatal stress contributes to wheeze directly and also through postnatal life events and respiratory infections.

\section{Introduction}

Asthma diagnosis is commonly preceded by childhood wheezing. In the U.S., $48.5 \%$ of children experience one or more wheezing episodes before age six years ${ }^{1}$. There is growing interest in perinatal factors related to wheeze/asthma since better understanding can suggest opportunities for early prevention. In-utero exposure to psychosocial stress has recently been advanced as a potential risk ${ }^{2}$. In a meta-analysis of ten studies, perceived stress, depression, anxiety, and adverse life events during pregnancy were significant predictors of 
paediatric wheeze/asthma ${ }^{3}$. Stress hormones cross placenta, influencing foetal development of diverse organs and systems including the respiratory tract. Based on animal and human studies, biological mechanisms triggered by foetal stress exposure involve hypothalamic-pituitary-adrenal axis, autonomic nervous system, and immune system, affecting the development of immune and respiratory structure-function ${ }^{4,5}$, maternalfoetal immune interactions ${ }^{6}$, pro-inflammatory states $^{4}$, glucocorticoid action and sympathovagal balance ${ }^{7}$, elevated IgE levels in cord blood $^{8}$, and Th2-biased cell differentiation ${ }^{9}$.

Psychosocial stress during postpartum is important as well. Stressful life circumstances may compromise mother-infant interaction, disrupt childcare routines, and worsen healthcare access. Some types of stressful events, such as job loss or chronic illness/disability, can reduce family income, require accepting poorerquality housing, and leading to exposure to environmental pollutants and allergens, such as mold ${ }^{10}$. Breastfeeding benefits immune development and lowers the risk of allergic diseases ${ }^{11}$, but women who report more stress in their lives are less likely to breastfeed ${ }^{12}$.

Caution is needed when interpreting correlations between perinatal stress and wheeze/asthma. First, experimental evidence substantiating causal interpretation is generally limited to animal models. Pregnant mice stressed by sound, for instance, are more likely to have offspring with allergen-induced airway inflammation ${ }^{13}$. Human studies of stress during pregnancy typically rely on observation, making it less clear whether the observed relationships are causal or result from residual confounding ${ }^{9}$. Second, the quality of data varies. In a meta-analysis focusing on prenatal stress and allergic/atopic conditions ${ }^{9}$, only $15 \%$ of qualifying studies were deemed high-quality. Third, few investigations assess stress using instruments designed and validated for pregnant women. Research on psychosocial stress in humans more generally is fraught with methodological weaknesses ${ }^{14}$. A conceptual definition of stress is often lacking, making it difficult to evaluate conceptmeasure fit. Instead of direct stress measurement, studies often use proxy measures originally designed for other concepts, e.g., psychological distress, anxiety, and depression. In a meta-analysis ${ }^{3}, 60 \%$ of studies assessed stress during pregnancy using measures of depression, psychological distress, and self-reported or physician-diagnosed anxiety. This approach is problematic because of conceptual conflation ${ }^{14}$. Six prospective studies ${ }^{15-20}$ and one retrospective study ${ }^{21}$ of asthma/wheeze in children used a more direct approach, measuring stress through exposure to life events.

A notable limitation is the lack of a theoretical framework in most research on perinatal stress and wheezing. To address this gap, we build on recent scholarship to propose a conceptual model displayed in Figure 1. Life events during pregnancy are conceptualized as psychosocial stressors that lead to maternal stress response. Maternal stress hormones crossing placenta contribute to immune, endocrine, and respiratory dysregulation in the foetus ${ }^{4,5}$, especially when stress is severe and long-lasting. Some types of psychosocial stressors tend to persist long-term and proliferate across life domains, generating yet new stressful circumstances for the mother and child ${ }^{22}$. Job loss in the family, for instance, can lead to a cascade of stressful events, e.g., job search, residential move, and changes in lifestyle to accommodate income loss. Consequently, we expect higher stress among postpartum women who had experienced high stress when pregnant. Postnatal stress adversely influences maternal health behaviour and mother-infant interaction. Hence, postnatal stress is conceptualized as a mediator between prenatal stress and wheeze. Additional mediators include lower respiratory tract infections (LRTI) ${ }^{23}$, allergies, lack of breastfeeding ${ }^{11}$, and secondary smoke exposure $(\mathrm{SSE})^{24}$. These mediators are selected since in past literature, they relate both to wheeze and to perinatal stress. Higher perceived stress, for instance, contributes to tobacco smoking ${ }^{25}$ and prenatal stress is linked to allergic rhinitis, atopic eczema/dermatitis, and infectious diseases in offspring ${ }^{26}$.

Figure 1

The following testable hypotheses are derived from the proposed model:

H1: Higher exposure to prenatal life events relates to higher risk of childhood wheeze.

H2: Higher exposure to postnatal life events relates to higher risk of childhood wheeze.

H3: The relationship between prenatal life events and wheeze is mediated by postnatal life events (H3a), 
smoking in pregnancy (H3b), SSE (H3c), lack/short duration of breastfeeding (H3d), LRTI (H3e), and allergy (H3f).

H4: The relationship between postnatal life events and wheeze is mediated by SSE (H4a), lack/short duration of breastfeeding (H4b), LRTI (H4c), and allergy (H3d).

These hypotheses are tested using prospective population-based data. Addressing limitations of prior research, stress assessment is based on exposure to life events using an instrument specifically designed for pregnancy/postpartum. A range of life events is covered for comprehensive assessment of psychosocial stress, including events with unique relevance for pregnancy/postpartum, e.g., learning about pregnancy complications. The size of the analytical sample $(n=1,849)$ ensures an adequate number of cases of child wheeze, facilitating multivariate modelling and mediation analysis.

\section{Methods}

Data

Data are obtained from European Longitudinal Cohort Study of Pregnancy and Childhood-Czech Republic (ELSPAC-CZ). The study was initiated by World Health Organization to investigate factors in maternal and child health in several European countries. The Czech part was approved for adherence to ethical guidelines by the Scientific Council of Masaryk University. The Czech cohort targeted the entire population of births in two cities between March 1, 1991 and June 30, 1992 ${ }^{27}$. Brno, a large metropolis and Znojmo, a smaller nearby town, were chosen to represent urban and rural populations. Medical documentation was available for 7,589 births ( $96 \%$ of all eligible births). To assess prenatal characteristics, the study partnered with local obstetrics/gynecology practices to distribute survey questionnaires to gravidas at 20 weeks (henceforth mid-pregnancy). This effort yielded 4,811 responses for the baseline survey sample. All participating women provided written informed consent. When the focal child reached age six months, eighteen months, three years, five years, and seven years, women were mailed follow-up surveys on child's health. At child age seven years, 3,312 mother-child pairs remained in the cohort for $67 \%$ of the baseline sample. Of these, 1,849 mother-child pairs had complete data and are included in the analytical sample.

Measures

The main outcome, child's wheeze/wheeze with whistle (henceforth "wheeze"), was collected at age six months, eighteen months, three years, five years, and seven years. At each time point, mothers were asked, "Were there any periods [between observation time points] when there was wheezing or wheezing with whistling on your child's chest when breathing?" (yes/no). Following Tucson Children's Respiratory Study (TCRS ${ }^{28,29}$, wheeze was coded as four longitudinal phenotypes, including "never" (no wheezing at any time point), "early-onset transient" (EOT) for onset before age three years with resolution by age seven years, "earlyonset persistent" (EOP) for onset before age three years persisting at age seven years, and "late-onset" (LO) for onset between age three years and seven years. While TCRS used age six years as a cut-off, we use seven years as the closest available observation point.

Prenatal and postnatal life events, main predictors, were assessed using Social Readjustment Scale ${ }^{30}$ adapted for Avon Longitudinal Study of Pregnancy and Childhood. The questionnaire, tailored specifically for pregnancy and postpartum and used in prior population-based research ${ }^{31-33}$ lists 42 types of life events (Figure A1 and A2 in SI). Events range from rare, e.g., death of a spouse, to more common, e.g., problems at work. Respondents report whether each event occurred during a specified period. Prenatal stress was assessed in mid-pregnancy using events that occurred since conception. Postnatal stress was assessed at six months postpartum using events that occurred since the delivery. For each observation period, stress exposure was represented by the total number of reported events categorized as low, medium, and high using terciles.

Mediators. To represent no/short breastfeeding as a risk factor, we used maternal reports of breastfeeding duration collected at eighteen months; for non-responding mothers, breastfeeding duration was extracted from paediatric records. The duration was dichotomized using the cut-off of four months, the approximate mean. Mothers reporting no breastfeeding or breastfeeding $<4$ months were coded as 1 for no/short 
breastfeeding; others were coded as 0 .

LRTI and allergy diagnoses for age 0-18 months were extracted from paediatric documentation. Both variables were coded 1 for children with the diagnosis and 0 for others.

Current maternal smoking (yes/no) was self-reported in mid-pregnancy.

Child's SSE was mother-reported at eighteen months, coded as hours per week, and treated as continuous.

Covariates. Demographic background includes maternal education (years), marital status (married vs. non-married), age at delivery (years), child sex (male vs. female), and residential region (Brno vs. Znojmo). Additional covariates, selected using literature on paediatric wheeze/asthma, include child allergy extracted from medical records at age eighteen months, maternal histories of asthma and allergy ${ }^{34}$ self-reported in mid-pregnancy, and low birth weight $(<2,500 \text { grams })^{35}$ extracted from obstetric documentation. Parity and singleton birth are used as proxies for siblings in the household ${ }^{11}$.

Statistical Analysis

Analysis was conducted using Stata 14.0 statistical software. Descriptive statistics were obtained including frequencies, percentages, means, and standard deviations. Bivariate relationships between life events and wheeze phenotypes were assessed using simple multinomial logistic regression models. Next, full multivariate models were fitted including all hypothesized predictors, mediators (prenatal life events, postnatal life events, LRTI, allergy, smoking in pregnancy, SSE, breastfeeding), demographic characteristics (child sex, maternal age and its square, education, region, singleton birth), and all remaining covariates. Final models testing $\mathrm{H} 1$ and $\mathrm{H} 2$ retained hypothesized predictors, mediators, and demographic characteristics; other covariates were trimmed for parsimony to those with $\mathrm{p}<0.10$ in the full model. These included parity and maternal asthma history. Predicted probabilities for each wheeze phenotype were obtained using marginal effects. Finally, mediation analysis was conducted to test $\mathrm{H} 3$ and $\mathrm{H} 4$. Wheeze was dichotomized as no wheeze vs. any wheeze in mediation analysis since methodology for mediation with dichotomous outcomes is better developed compared to multi-category outcomes ${ }^{36}$. Decomposition of total effects into direct and indirect was performed using "ldecomp" command ${ }^{37}$ with 1,000 bootstrap replications to produce $95 \%$ bias-corrected confidence intervals.

\section{Results}

The analytical sample included 879 boys and 970 girls. By age seven years, $37.6 \%$ of children experienced one or more wheezing episodes (Table 1). EOT wheeze was the most common phenotype (20.5\%); followed by LO (9.3\%) and EOP (7.8\%). Paediatrician-diagnosed LRTIs were common (46.8\%). Every fifth mother reported smoking during pregnancy $(19.6 \%)$ and $30.8 \%$ reported that their toddler had some exposure to tobacco smoke. Majority of children $(80.8 \%)$ were breastfed; over a half $(53.5 \%)$ for less than four months.

Table 1

Wheezing was linked to smoking during pregnancy $(\mathrm{p}=0.044)$, child's SSE $(\mathrm{p}=0.008)$, LRTI $(\mathrm{p}<0.001)$, and lack/short duration of breastfeeding $(\mathrm{p}=0.035)$. Wheezing was more common among boys $(\mathrm{p}<0.001)$, Brno residents $(\mathrm{p}=0.031)$, second-born and higher birth-order children $(\mathrm{p}=0.044)$, and children of mothers with a history of asthma $(\mathrm{p}=0.028)$.

In bivariate models showing unadjusted relationships, the relative risk of wheezing vs. no wheezing was 54\%-79\% higher among children with high vs. low exposure to prenatal life events (Table 2). Relative risk ratios (RRR) for wheeze phenotypes were 1.54 (95\% CI 1.16-2.05, $\mathrm{p}=0.003)$ for EOT; 1.72 (95\% CI 1.13-2.63, $\mathrm{p}=0.012)$ for EOP; and $1.79(95 \%$ CI 1.22-2.61, $\mathrm{p}=0.003)$ for LO. Increases in the relative risk of wheeze linked to postnatal life events were even higher. High vs. low exposure was associated with 57\%-145\% increases $(\mathrm{EOT}, \mathrm{RRR}=2.11,95 \%$ CI 1.58-2.82, $\mathrm{p}<0.001$; $\mathrm{EOP}, \mathrm{RRR}=2.45,95 \%$ CI 1.55-3.90, $\mathrm{p}<0.001 ; \mathrm{LO}$, $\mathrm{RRR}=1.57,95 \%$ CI $1.06-2.32, \mathrm{p}=0.026)$.

Table 2 


\section{Multivariate Results}

Adjusted Relationships between Perinatal Life Events and Wheeze.In Table 2, statistically significant differences in the likelihood of several wheeze phenotypes by high vs. low exposure to life events remain evident even after adjustment for covariates. Children with highprenatal exposure to life events have a $44 \%$ higher relative risk of EOT wheeze compared to counterparts with low exposure $(\mathrm{RRR}=1.44,95 \% \mathrm{CI}$ 1.06-1.95, $\mathrm{p}=0.020)$. Moreover, they have a $69 \%$ higher relative risk of $\mathrm{LO}$ wheeze $(\mathrm{RRR}=1.69,95 \% \mathrm{CI}$ 1.13-2.53, $\mathrm{p}=0.010$ ). EOP wheeze approaches significance with RRR of 1.56 (95\% CI 0.99-2.44, $\mathrm{p}=0.053$ ). For postnatal life events, high vs. low exposure is related to a $74 \%$ increase in relative risk of EOT wheeze $(\mathrm{RRR}=1.74,95 \%$ CI 1.28-2.36, $\mathrm{p}<0.001)$; the corresponding increase for EOP wheeze is $101 \%(\mathrm{RRR}=2.01$, 95\% CI 1.23-3.26, $\mathrm{p}=0.005)$. These results support $\mathrm{H} 1$ and $\mathrm{H} 2$.

Figure 2 illustrates predicted probabilities of each wheeze phenotype based on marginal effects calculated for four categories of stress exposure, overall low stress (low prenatal/low postnatal life events); increasing stress (low prenatal/high postnatal life events); decreasing stress (high prenatal/low postnatal life events); and overall high stress (high prenatal/high postnatal life events). In all stress categories, the majority of children never wheeze, but the probability of staying wheeze-free throughout the observation period is considerably lower among children with overall high stress $(51.9 \%)$ vs. children with overall low stress $(72.2 \%)$. The predicted probability of EOT wheeze more than doubles among children in the overall high stress category $(9.4 \%)$ compared to overall low stress category $(4.5 \%)$ Predicted probabilities corresponding to overall low stress vs. overall high stress are $15.4 \%$ vs. $26.2 \%$, respectively, for EOT wheeze and $8.0 \%$ vs. $12.5 \%$, respectively, for $\mathrm{LO}$ wheeze.

Figure 2

Mediation between Prenatal Life Events and Wheeze. Models of mediators are displayed in Table 3. Unstandardized coefficients are reported for all types of models to facilitate interpretation. Higher prenatal life events predict higher postnatal life events (high vs. low prenatal life events, unstandardized coefficient $\mathrm{b}=0.77,95 \%$ CI 0.49-1.06, $\mathrm{p}<0.001$ ). Decomposition analysis reveals that postnatal life events partially mediate between prenatal life events on any wheeze vs. no wheeze (high vs. low prenatal events, direct effect odds ratio $[\mathrm{OR}]=1.51,95 \%$ CI 1.18-1.93, $\mathrm{p}=0.001$; indirect effect $\mathrm{OR}=1.13,95 \%$ CI 1.06-1.21, $\mathrm{p}<0.001$; total effect $\mathrm{OR}=1.7195 \%$ CI 1.34-2.18, $\mathrm{p}<0.001$ ), supporting H3a. Prenatal life events are unrelated to smoking during pregnancy, SSE, breastfeeding, LTRI, and allergy, ruling out mediation through these factors.

Table 3

Mediation between Postnatal Life Events and Wheeze. Children with higher exposure to postnatal life events are more commonly diagnosed with LRTI (Table 2) and LRTI mediates between postnatal life events and any wheeze (high vs. low postnatal events, direct effect $\mathrm{OR}=1.85,95 \%$ CI $0.05-74.31, \mathrm{p}=0.745$; indirect effect $\mathrm{OR}=1.06,95 \%$ CI 1.02-1.09, $\mathrm{p}=0.002$; total effect OR 1.95, 95\% CI 0.05-78.75, $\mathrm{p}=0.723$ ). This result supports H4c. Postnatal life events are also linked to SSE, another significant mediator (direct effect $\mathrm{OR}=1.86,95 \%$ CI $0.04-82.06, \mathrm{p}=0.749$; indirect effect $\mathrm{OR}=1.02,95 \%$ CI 1.001-1.05, $\mathrm{p}=0.035)$. Mediation through breastfeeding is unsupported.

To illustrate multivariate results, Figure 3 displays estimated statistical effects for each pathway in the proposed conceptual model. It demonstrates the mediation of prenatal stress through postnatal stress, mediation of postnatal stress through respiratory infections, and mediation of postnatal stress through SSE.

Figure 3 here

Covariate Effects (results upon request). In the final multinomial logistic regression model of wheeze phenotypes, boys are more likely to develop early-onset wheeze compared to girls (EOT, RRR=1.60; 95\% CI 1.25-2.04, $\mathrm{p}<.001$; $\mathrm{EOP}, \mathrm{RRR}=1.61$; CI 1.13-2.31, $\mathrm{p}=0.009)$. EOP wheeze is related to maternal asthma history $(\mathrm{RRR}=3.08,95 \%$ CI 1.44-6.58, $\mathrm{p}=0.004)$.

\section{Discussion}


To our knowledge, this is the first prospective, population-based, birth-cohort study considering wheeze phenotypes during early to mid-childhood in relation to stress assessed as life events at different time points during perinatum. Prior studies rarely distinguished among longitudinal phenotypes of wheeze and typically measured stress indirectly as psychological distress. The present study documents that early exposure to stressful events is linked to wheezing. The timing of stress exposure matters for which wheeze phenotype likely develops. The likelihood of wheeze with late onset increases with stress exposure during pregnancy. Stress exposure during the first six months after birth, in contrast, predicts persistent wheeze, which manifests by age three years and persists until age seven years or longer. In prior literature, persistent wheeze showed a relationship with poor lung function and chronic asthma ${ }^{38}$, warranting better understanding of this particular wheeze phenotype.

Specifying pathways leading from maternal stress to child wheezing represents a contribution since these pathways were rarely examined in prior literature. Perhaps without surprise is the finding that women who experience more stress during pregnancy also tend to experience more stress after delivery, which accounts in part for the link between prenatal stress and wheezing. More intriguing is the finding that mediation through postnatal stress is partial only, leaving an independent effect of prenatal stress of a substantial magnitude, with the increase in relative risk ranging 44\%-69\%. We examined several additional mechanisms from perinatal stress to wheezing. LRTI diagnosis emerged as a mediator, suggesting that children exposed to more postnatal stress are more likely to develop LRTIs. This accounts for some, but not for all, of the increase in wheezing among children of mothers exposed to more postnatal life events. In other words, the significant association between early-life stress exposure and wheezing persists after adjusting for LRTI and covariates.

Multivariate modelling strengthens the evidence of relationships independent of confounders, but causal interpretation is not warranted with observational data. Nevertheless, using rigorous statistical controls and mediation analysis, we rule out several alternative mechanisms. This is an important contribution since mechanisms for the stress gradient in wheezing are under-explored. Maternal emotional dysregulation, for instance, is a candidate mechanism. It is commonly preceded by stress exposure. Literature suggests that maternal emotional difficulties are linked to child respiratory problems ${ }^{3}$. Other mechanisms warranting future research include neuro-endocrine-immune processes involved in airway functioning.

Childhood wheeze is commonly discussed in literature on allergic asthma. Allergic asthma bronchiale is defined by allergic eosinophil inflammation with bronchial obstruction. Wheeze is its symptom but it is also a broader nosologic unit with heterogeneous pathogenic origins. Factors triggering wheeze are not limited to allergic reaction; they also include infection, irritation, and sudden change in ambient temperature. Prior analysis of ELSPAC-CZ found that children with persistent wheeze had higher risk of allergic asthma during school age ${ }^{38}$. Surprising, our supplementary analyses indicated that allergic asthma diagnosis at ages five and fifteen years did not relate to prenatal or postnatal life events in adjusted models (five years, high vs. low prenatal life events odds ratio $[\mathrm{OR}]=2.63,95 \%$ CI $0.74-9.34, \mathrm{p}=0.113$; five years, high vs. low postnatal life events $\mathrm{OR}=0.71,95 \%$ CI $0.22-2.26, \mathrm{p}=0.653$; fifteen years, high vs. low prenatal life events $\mathrm{OR}=1.01$, 95\% CI 0.59-1.73, $\mathrm{p}=0.938$; fifteen years, high vs. low postnatal life events $\mathrm{OR}=0.99,95 \%$ CI $0.57-1.73$, $\mathrm{p}=0.975$ ). Moreover, neither prenatal nor postnatal life events were associated with physician-diagnosed allergy among children ages 0-18 months, as indicated in Figure 3. We speculate that early-life stress is important for the types of wheeze unrelated to allergic asthma. Our data did not distinguish between allergic vs. non-allergic wheeze; mothers simply reported whether their child experienced any wheezing episodes. Yet, the observation that LRTI partially mediated between life events and wheeze suggests the involvement of respiratory infections, potentially accounting for some cases of wheeze unrelated to allergy, though this explanation is tentative.

Strengths of this study include prospective design with a large sample of mother-child dyads with fully completed questionnaires $(\mathrm{n}=1,849)$; accounting for multiple mechanisms and confounders; using established methodology to categorize wheeze phenotypes ${ }^{28}$, and separately considering stress exposures during prenatal vs. postnatal periods. Both periods are developmentally sensitive but they differ in the types of developmen- 
tal processes taking place. Assessment of life events was quite broad with 42 different types of events, yielding a more comprehensive picture of the overall stress levels compared to more narrowly-focused instruments.

Methodological limitations must be noted. The cohort included mostly young gravidas with uncomplicated pregnancies. Mean age at delivery (25.2 years) and the caesarean section rate (8.1\%) roughly correspond to the demographic profile of the Czech population at the time of the study ${ }^{39}$, suggesting good generalizability except for older and complicated gravidas. Generalizability to societies outside of the Czech Republic, however, is unknown. Child wheeze was mother-reported. Maternal reports are routinely used in epidemiological surveys of child health ${ }^{40}$. They are cost-effective and capture symptoms known only to the mother, such as wheezing episodes for which healthcare was not obtained. Potential biases include under-report (child wheezed; mother reported no wheezing), over-report (child did not wheeze; mother reported wheezing), and misreport (child wheezed; mother reported another condition). Finally, biomarkers quantifying biological mechanisms between early-life stress and wheeze were not available.

\section{Conclusion}

This study proposes a conceptual model linking psychosocial stress during the prenatal and postnatal periods to respiratory problems in children, testing the model with prospective evidence. The supported mechanisms through which perinatal stress exposure contributes to childhood wheeze include LTRI and SSE. These findings represent a step toward better understanding of early-life origins of wheezing though the exact aetiology remains unknown. More precise identification of sources of respiratory health risks in children can inform early preventive efforts targeting women and children at risk during critical periods of development.

\section{Acknowledgements}

The authors of this study wish to thank the participating families as well as the gynaecologists, paediatricians, school heads, and class teachers who took part in the project. Our thanks also go to Dr. Lubomír Kukla, Ph.D., ELSPAC national coordinator 1990-2012, and the entire ELSPAC team. The authors of this study (i.e. not the ELSPAC Scientific Council) are responsible for the contents of this publication.

\section{Conflict of Interest}

Dr. Stepanikova has nothing to disclose. Dr. Mikes has nothing to disclose. Dr. Thon has nothing to disclose. Dr. Klanova has nothing to disclose.

\section{References}

1. Martinez FD, Wright AL, Taussig LM, Holberg CJ, Halonen M, Morgan WJ. Asthma and wheezing in the first six years of life. New England Journal of Medicine. 1995;332(3):133-138. doi:10.1056/NEJM199501193320301

2. Lee A, Wright RJ. Prenatal stress and childhood asthma risk: Taking a broader view. European Respiratory Journal. 2016 [accessed 2020 Mar 11];47(2):406-409. http://www.ncbi.nlm.nih.gov/pubmed/26828054. doi:10.1183/13993003.01921-2015

3. Van De Loo KFE, Van Gelder MHJ, Roukema J, Roeleveld N, Merkus PJFM, Verhaak CM. Prenatal maternal psychological stress and childhood asthma and wheezing: A meta-analysis. European Respiratory Journal. 2016;47(1):133-146. doi:10.1183/13993003.00299-2015

4. Prescott SL. The development of respiratory inflammation in children. Paediatric Respiratory Reviews. 2006;7(2):89-96. doi:10.1016/j.prrv.2006.03.001

5. Elenkov IJ, Kvetnansky R, Hashiramoto A, Bakalov VK, Link AA, Zachman K, Crane M, Jezova D, Rovensky J, Dimitrov MA, et al. Low- versus High-Baseline Epinephrine Output Shapes Opposite Innate Cytokine Profiles: Presence of Lewis- and Fischer-Like Neurohormonal Immune Phenotypes in Humans? The Journal of Immunology. 2008;181(3):1737-1745. doi:10.4049/jimmunol.181.3.1737 
6. Lim RH, Kobzik L. REVIEW ARTICLE: Maternal Transmission of Asthma Risk. American Journal of Reproductive Immunology. 2008 [accessed 2019 Dec 3];61(1):1-10. http://doi.wiley.com/10.1111/j.16000897.2008.00671.x. doi:10.1111/j.1600-0897.2008.00671.x

7. Buijs RM, van der Vliet J, Garidou M-L, Huitinga I, Escobar C. Spleen Vagal Denervation Inhibits the Production of Antibodies to Circulating Antigens Akbarian S, editor. PLoS ONE. 2008 [accessed 2019 Dec 3];3(9):e3152. https://dx.plos.org/10.1371/journal.pone.0003152. doi:10.1371/journal.pone.0003152

8. Sternthal MJ, Enlow MB, Cohen S, Canner MJ, Staudenmayer J, Tsang K, Wright RJ. Maternal interpersonal trauma and cord blood IgE levels in an inner-city cohort: A life-course perspective. Journal of Allergy and Clinical Immunology. 2009;124(5):954-960. doi:10.1016/j.jaci.2009.07.030

9. Flanigan C, Sheikh A, DunnGalvin A, Brew BK, Almqvist C, Nwaru BI. Prenatal maternal psychosocial stress and offspring's asthma and allergic disease: A systematic review and meta-analysis. Clinical and Experimental Allergy. 2018;48(4):403-414. doi:10.1111/cea.13091

10. Kranjac AW, Kimbro RT, Denney JT, Osiecki KM, Moffett BS, Lopez KN. Comprehensive Neighborhood Portraits and Child Asthma Disparities. Maternal and Child Health Journal. 2017 [accessed 2020 Mar 11];21(7):1552-1562. http://www.ncbi.nlm.nih.gov/pubmed/28181157. doi:10.1007/s10995-017-2286-z

11. Just J, Belfar S, Wanin S, Pribil C, Grimfeld A, Duru G. Impact of innate and environmental factors on wheezing persistence during childhood. Journal of Asthma. 2010;47(4):412-416. doi:10.3109/02770900903584035

12. Groër MW. Differences between exclusive breastfeeders, formula-feeders, and controls: A study of stress, mood, and endocrine variables. Biological Research for Nursing. 2005;7(2):106-117. doi: $10.1177 / 1099800405280936$

13. Quarcoo D, Pavlovic S, Joachim RA. Stress and airway reactivity in a murine model of allergic airway inflammation. NeuroImmunoModulation. 2009;16(5):318-324. doi:10.1159/000216189

14. Slavich GM. Stressnology: The primitive (and problematic) study of life stress exposure and pressing need for better measurement. Brain, Behavior, and Immunity. 2019 [accessed 2020 Mar 11];75:3-5. http://www.ncbi.nlm.nih.gov/pubmed/30236597. doi:10.1016/j.bbi.2018.08.011

15. Bandoli G, von Ehrenstein O, Ghosh JKC, Flores MES, Dunkel Schetter C, Ritz B. Prenatal Maternal Stress and the Risk of Lifetime Wheeze in Young Offspring: An Examination by Stressor and Maternal Ethnicity. Journal of Immigrant and Minority Health. 2016;18(5):987-995. doi:10.1007/s10903-015-0269-y

16. Chiu YHM, Coull BA, Cohen S, Wooley A, Wright RJ. Prenatal and postnatal maternal stress and wheeze in urban children: Effect of maternal sensitization. American Journal of Respiratory and Critical Care Medicine. 2012;186(2):147-154. doi:10.1164/rccm.201201-0162OC

17. Lee A, Mathilda Chiu YH, Rosa MJ, Jara C, Wright RO, Coull BA, Wright RJ. Prenatal and postnatal stress and asthma in children: Temporal- and sex-specific associations. Journal of Allergy and Clinical Immunology. 2016;138(3):740-747.e3. doi:10.1016/j.jaci.2016.01.014

18. Rosa MJ, Just AC, Tamayo Y Ortiz M, Schnaas L, Svensson K, Wright RO, Téllez Rojo MM, Wright RJ. Prenatal and postnatal stress and wheeze in Mexican children: Sex-specific differences. Annals of Allergy, Asthma and Immunology. 2016;116(4):306-312.e1. doi:10.1016/j.anai.2015.12.025

19. Turcotte-Tremblay AM, Lim R, Laplante DP, Kobzik L, Brunet A, King S. Prenatal maternal stress predicts childhood asthma in girls: Project ice storm. BioMed Research International. 2014;2014. doi: $10.1155 / 2014 / 201717$

20. Hartwig IRV, Sly PD, Schmidt LA, Van Lieshout RJ, Bienenstock J, Holt PG, Arck PC. Prenatal adverse life events increase the risk for atopic diseases in children, which is enhanced in the absence of a maternal atopic predisposition. Journal of Allergy and Clinical Immunology. 2014;134(1). doi:10.1016/j.jaci.2014.01.033 
21. De Marco R, Pesce G, Girardi P, Marchetti P, Rava M, Ricci P, Marcon A. Foetal exposure to maternal stressful events increases the risk of having asthma and atopic diseases in childhood. Pediatric Allergy and Immunology. 2012;23(8):724-729. doi:10.1111/j.1399-3038.2012.01346.x

22. Pearlin LI. The life course and the stress process: Some conceptual comparisons. Journals of Gerontology - Series B Psychological Sciences and Social Sciences. 2010;65 B(2):207-215. doi:10.1093/geronb/gbp106

23. Silva-Estrada J, Reyna-Figueroa J, Wakida-Kusonoki G, Limón-Rojas A, Campos-Romero F. Wheezing Disorders in Childhood. Clinical Case Reports International. 2018 [accessed 2019 Dec 4];2:1038. http://www.clinicalcasereportsint.com/full-text/ccri-v2-id1038.php

24. Vardavas CI, Hohmann C, Patelarou E, Martinez D, Henderson AJ, Granell R, Sunyer J, Torrent M, Fantini MP, Gori D, et al. The independent role of prenatal and postnatal exposure to active and passive smoking on the development of early wheeze in children. European Respiratory Journal. 2016;48(1):115-124. doi:10.1183/13993003.01016-2015

25. Damron KR. Review of the Relationships Among Psychosocial Stress, Secondhand Smoke, and Perinatal Smoking. JOGNN - Journal of Obstetric, Gynecologic, and Neonatal Nursing. 2017;46(3):325-333. doi:10.1016/j.jogn.2017.01.012

26. Stepanikova I, Baker E, Oates G, Acharya S, Uddin J, Thon V, Svancara J, Kukla L. Perinatal Maternal Stress and Susceptibility to Infectious Diseases in Later Childhood: An Early Life Programming Perspective. Journal of Psychology: Interdisciplinary and Applied. 2019;153(1):67-88. doi:10.1080/00223980.2018.1483311

27. Pavel Piler; Vít Kandrnal; Lubomír Kukla; Lenka Andrýsková; Jan Švancara; Jiří Jarkovský; Ladislav Dušek; Hynek Pikhart; Martin Bobák; Jana Klánová. Cohort profile: European Longitudinal Study of Pregnancy and Childhood (ELSPAC) in the Czech Republic. International Journal of Epidemiology. 2016. doi:10.1093/ije/dyw091

28. Taussig LM, Wright AL, Holberg CJ, Halonen M, Morgan WJ, Martinez FD. Tucson children's respiratory study: 1980 to present. Journal of Allergy and Clinical Immunology. 2003 [accessed 2017 Jun 21];111(4):661675. http://linkinghub.elsevier.com/retrieve/pii/S0091674902914818. doi:10.1067/mai.2003.162

29. Morgan WJ, Stern DA, Sherrill DL, Guerra S, Holberg CJ, Guilbert TW, Taussig LM, Wright AL, Martinez FD. Outcome of asthma and wheezing in the first 6 years of life follow-up through adolescence. American Journal of Respiratory and Critical Care Medicine. 2005;172(10):1253-1258. doi:10.1164/rccm.200504-525OC

30. Holmes TH, Rahe RH. The social readjustment rating scale. Journal of Psychosomatic Research. 1967;11(2):213-218. doi:10.1016/0022-3999(67)90010-4

31. Dorrington S, Zavos H, Ball H, McGuffin P, Rijsdijk F, Siribaddana S, Sumathipala A, Hotopf M. Trauma, post-traumatic stress disorder and psychiatric disorders in a middle-income setting: Prevalence and comorbidity. British Journal of Psychiatry. 2014;205(5):383-389. doi:10.1192/bjp.bp.113.141796

32. Hibbeln JR, Davis JM, Steer C, Emmett P, Rogers I, Williams C, Golding J. Maternal seafood consumption in pregnancy and neurodevelopmental outcomes in childhood (ALSPAC study): an observational cohort study. Lancet. 2007;369(9561):578-585. doi:10.1016/S0140-6736(07)60277-3

33. Sullivan S, Rai D, Golding J, Zammit S, Steer C. The Association Between Autism Spectrum Disorder and Psychotic Experiences in the Avon Longitudinal Study of Parents and Children (ALSPAC) Birth Cohort. Journal of the American Academy of Child \& Adolescent Psychiatry. 2013 [accessed 2019 Dec 4];52(8):806814.e2. https://linkinghub.elsevier.com/retrieve/pii/S0890856713003377. doi:10.1016/j.jaac.2013.05.010

34. Rodríguez-Martínez CE, Sossa-Briceño MP, Castro-Rodriguez JA. Factors predicting persistence of early wheezing through childhood and adolescence: A systematic review of the literature. Journal of Asthma and Allergy. 2017;10:83-98. doi:10.2147/JAA.S128319 
35. Leung JYY, Lam HS, Leung GM, Schooling CM. Gestational Age, Birthweight for Gestational Age, and Childhood Hospitalisations for Asthma and Other Wheezing Disorders. Paediatric and Perinatal Epidemiology. 2016;30(2):149-159. doi:10.1111/ppe.12273

36. Iacobucci D. Mediation analysis and categorical variables: The final frontier. Journal of Consumer Psychology. 2012;22(4):582-594. doi:10.1016/j.jcps.2012.03.006

37. Buis ML. Direct and indirect effects in a logit model. Stata Journal. 2010;10(1):11-29. doi:10.1177/1536867x1001000104

38. Mikeš O, Vrbová M, Klánová J, Čupr P, Švancara J, Pikhart H. Early-life exposure to household chemicals and wheezing in children. Science of the Total Environment. 2019;663:418-425. doi:10.1016/j.scitotenv.2019.01.254

39. Srp B, Velebil P. Proportion of caesarean sections and main causes of maternal mortality during 1978-1997 in the Czech Republic. Ceska gynekologie. 1999 [accessed 2020 Mar 11];64(4):219-23. http://www.ncbi.nlm.nih.gov/pubmed/10568057

40. McCormick MC, Athreya BH, Bernbaum JC, Charney EB. Preliminary observations on maternal rating of health of children: Data from three subspecialty clinics. Journal of Clinical Epidemiology. 1988 [accessed 2020 Mar 11];41(4):323-329. http://www.ncbi.nlm.nih.gov/pubmed/3351540. doi:10.1016/08954356(88)90139-4

\section{Hosted file}

Wheeze_Tables_submitted.docx available at https://authorea.com/users/345136/articles/471458a-model-of-perinatal-stress-and-childhood-wheezing-elspac-cz-cohort

\section{Hosted file}

Figures down.docx available at https://authorea.com/users/345136/articles/471458-a-model-ofperinatal-stress-and-childhood-wheezing-elspac-cz-cohort 